\title{
Instabilities of dispersion-managed solitons in the normal dispersion regime
}

\author{
Dmitry E. Pelinovsky* \\ Department of Mathematics, University of Toronto \\ Toronto, Ontario, Canada, M5S 3G3
}

October 27, 2018

\begin{abstract}
:
Dispersion-managed solitons are reviewed within a Gaussian variational approximation and an integral evolution model. In the normal regime of the dispersion map (when the averaged path dispersion is negative), there are two solitons of different pulse duration and energy at a fixed propagation constant. We show that the short soliton with a larger energy is linearly (exponentially) unstable. The other (long) soliton with a smaller energy is linearly stable but hits a resonance with excitations of the dispersion map. The results are compared with the results from the recent publications [3, 嵑.
\end{abstract}

PACS: $03.40 . K f$, 42.65.Tg, 42.81.Dp

Key words: dispersion management, optical solitons, instability.

*dmpeli@math.toronto.edu 


\section{Introduction}

New ways in optimization of existing telecommunication systems based on dispersion management technology attracted recently wide research interest from soliton-based groups (see reviews [1, 21). The main idea was to combine a high local group-velocity dispersion with a low path-average dispersion. The former feature results in the reduction of the four-wave mixing while the latter one reduces the Gordon-Haus timing jitter effects. When the path-average dispersion is small and normal, i.e. the defocussing segment in the fiber is dominant over the focussing one, a new phenomenon of branching of soliton solutions was discovered [3, 4, 5, 6, 7]. The soliton propagation in this regime is not supported by an uniform-dispersion optical fiber and seems to be one of the remarkable achievement of the dispersion management with sufficiently high local dispersion.

The stability of branching soliton solutions in the normal dispersion regime was a subject of intense studies which lead to contradictory conclusions. Grigoryan and Menyuk announced the linear and nonlinear stability of both the branches [5], while Berntson et al. conjectured instability of one of the branches [3].

In this paper, we intend to shed light on the complicated issue of existence and stability of soliton signals in the normal regime of the dispersion map. We find, in the small-amplitude approximation, that there exist two branches of soliton solutions for different levels of energy and different pulse durations at a fixed propagation constant. The short pulses with larger energy are proved to be linearly unstable, while the other (long) pulses with smaller energy are neutrally stable. We show that the transition from large-energy unstable solitons to the stable soliton signals occurs via long-term transient oscillations. The two branches of soliton solutions correspond to a single (small-energy) branch B in Fig. 1 of [5]. Depending on a normalization condition (see Section 4), this branch may be either stable or unstable.

Our strategy to develop the small-amplitude approximation is based on the combination of two analytical approaches: the Gaussian variational approximation and the integral evolution model.

The Gaussian variational approximation, being inaccurate in details, is still useful for a quick and rough analysis (see 8 for review and references). Also it was shown that the method can be extended to a rigorous Gauss-Hermite expansion of the basic model [9]. We improve the previous results summarized in [8] by deriving a new dynamical system from the variational equations of a Gaussian pulse. The system clearly displays the linear and nonlinear instability of the short Gaussian pulse with larger energy.

More rigorous analysis of the problem is based on the integral evolution model obtained by 
Gabitov and Turitsyn [10] and by Ablowitz and Biondini [11]. Although this model is more complicated from computational point of view (see recent papers 112, 13]), we managed to study numerically the construction of the linear spectrum of dispersion-managed solitons. Our results confirm the instability and transition scenarios predicted within the variational model. We also deduce from this model that the soliton signals in the normal regime of the dispersion map are in resonance with the wave continuum of linear excitations of the map. The resonance implies isually the generation of wave packets from stable pulses oscillating in time. The latter effects are beyond the accuracy of the analytical model and are left for further studies.

\section{Gaussian approximation: New dynamical model}

We study the NLS model in the dimensionless form [6],

$$
i u_{z}+\frac{1}{2} D(z) u_{t t}+\epsilon\left(\frac{1}{2} D_{0} u_{t t}+|u|^{2} u\right)=0
$$

where $u(z, t)$ is the envelope of an optical pulse in the retarded reference frame of the fiber. The small parameter $\epsilon$ measures the length of the dispersion's map and the inverse variance of the local dispersion. After normalization, $D_{0}$ and $D(z)$ are assumed to be of order of $\mathrm{O}(1)$, and

$$
\langle D\rangle=\int_{0}^{1} D(z) d z=0, \quad D(z+1)=D(z) .
$$

Further physical motivations for derivation and verification of the model (1) can be found in [1], 2]. Soliton-like optical pulses are solutions of the model in the form,

$$
u(z, t)=\psi(z, t) e^{i \epsilon \mu z}
$$

where $\mu$ is the propagation constant and $\psi(z, t)$ is a soliton profile satisfying the boundary conditions,

$$
\psi(z+1, t)=\psi(z, t)
$$

and

$$
\lim _{t \rightarrow \infty} \psi(z, t)=0
$$

One of the conventional approximation for soliton solutions of NLS-type equations is based on averaging the Gaussian anzatz in the Lagrangian density and further varying the Lagrangian density with respect to parameters of the Gaussian pulse (see [8] for review). The Gaussian approximation

is the first term of the Gauss-Hermite expansions when solving the NLS equation (1) in the limit 
$\epsilon \rightarrow 0$ [9]. We apply the Gaussian anzatz in the form,

$$
u(z, t)=c(z) \exp \left(-\frac{(\alpha(z)-2 i \beta(z))}{\alpha(z)^{2}+4 \beta(z)^{2}} t^{2}+i \phi(z)\right)
$$

Here the four parameters of the Gaussian pulse are: $c(z)$ - the amplitude, $\phi(z)$ - the gauge parameter, $\alpha(z)$ - the pulse duration, and $\beta(z)$ - the chirp. It was found that the four equations for variations of the Lagrangian density can be decoupled into a system for $\alpha(z)$ and $\beta(z)$ of the form,

$$
\begin{aligned}
& \frac{d \alpha}{d z}=\frac{4 \epsilon E \alpha^{5 / 2} \beta}{\left(\alpha^{2}+4 \beta^{2}\right)^{3 / 2}} \\
& \frac{d \beta}{d z}=D(z)+\epsilon\left(D_{0}-\frac{E \alpha^{3 / 2}\left(\alpha^{2}-4 \beta^{2}\right)}{2\left(\alpha^{2}+4 \beta^{2}\right)^{3 / 2}}\right) .
\end{aligned}
$$

The phase factor $\phi(z)$ is expressed in terms of $\alpha(z)$ and $\beta(z)$,

$$
\frac{d}{d z}\left(\phi+\frac{1}{2} \arctan \frac{2 \beta}{\alpha}\right)=\frac{\epsilon E \alpha^{1 / 2}\left(3 \alpha^{2}+20 \beta^{2}\right)}{4\left(\alpha^{2}+4 \beta^{2}\right)^{3 / 2}}
$$

while the amplitude $c(z)$ is given in terms of the input energy constant $E$ as

$$
E=\frac{\sqrt{\alpha^{2}+4 \beta^{2}}}{\sqrt{2 \alpha}} c^{2}=\frac{1}{\sqrt{\pi}} \int_{0}^{1} d z \int_{-\infty}^{\infty} d t|u|^{2}(z, t)>0 .
$$

The stationary pulse (3) - (5) corresponds to the periodic solutions of the system (7) and (8) in the form,

$$
\alpha(z+1)=\alpha(z), \quad \beta(z+1)=\beta(z), \quad \phi(z+1)=\phi(z)+\epsilon \mu .
$$

For simplicity, we study the periodic solutions in the limit $\epsilon \rightarrow 0$ by using a two-step dispersion map with zero average,

$$
D(z)= \begin{cases}D_{1}, & 0<z<L \\ D_{2}, & L<z<1\end{cases}
$$

where

$$
m=D_{1} L=-D_{2}(1-L)>0 \text {. }
$$

The asymptotic solution in the limit $\epsilon \rightarrow 0$ can be sought in the regular form,

$$
\alpha(z)=\alpha_{s}+\mathrm{O}(\epsilon), \quad \beta(z)=\int_{0}^{z} D\left(z^{\prime}\right) d z^{\prime}+\beta_{s}+\mathrm{O}(\epsilon)
$$

where $\alpha_{s}, \beta_{s}$ are constant. The periodic solutions appear when $\beta_{s}=-m / 2$ and $\alpha_{s}$ is a root of the equation,

$$
D_{0}=E \alpha_{s}^{3 / 2}\left[\frac{1}{\left(m^{2}+\alpha_{s}^{2}\right)^{1 / 2}}-\frac{1}{2 m} \log \left(\frac{m+\left(m^{2}+\alpha_{s}^{2}\right)^{1 / 2}}{\alpha_{s}}\right)\right] .
$$


In addition, the propagation constant $\mu$ can be obtained as a function of $E$ and $\alpha_{s}$ according to the equation,

$$
\mu=\frac{1}{4} E \alpha_{s}^{1 / 2}\left[\frac{-2}{\left(m^{2}+\alpha_{s}^{2}\right)^{1 / 2}}+\frac{5}{m} \log \left(\frac{m+\left(m^{2}+\alpha_{s}^{2}\right)^{1 / 2}}{\alpha_{s}}\right)\right] .
$$

These equations have been already derived in the literature, see [6, [7] for (13) and [9] for (14). However, the relations (13) and (14) were viewed typically under the normalization condition,

$$
m=1, \quad E=\frac{1}{\sqrt{2 S}}, \quad \alpha_{s}=\frac{1}{S},
$$

where $S$ is called the map strength. In this normalization, the expression (13) gives a smallamplitude limit of the results of [3, 5], i.e. the slope $E / D_{0}$ is a function of $S$. The existence of solitons was identified both for $D_{0}>0$ (when $S<S_{t h r}$ ) and for $D_{0}<0$ (when $S<S_{t h r}$ ), where $S_{t h r} \approx 3.32$.

In this paper, we develop a different frame to view the soliton solutions (13) and (14). Guided by the stability analysis of solitons in generalized NLS equations [14], we fix the parameters of the model $\left(D_{0}, m\right)$ and construct periodic solutions as a one-parameter family in terms of the propagation constant $\mu$. As a result, the parameters $\alpha$ and $E$ can be found from (13)-(14) as $\alpha_{s}=\alpha_{s}(\mu)$ and $E=E(\mu)$. These functions are shown in Fig. 1(a,b) for $D_{0}=0.02$ and $m=2$ and in Fig. 2(a,b) for $D_{0}=-0.02$ and $m=2$. Obviously, the branching occurs at $D_{0}<0$ (i.e. at the normal regime of the dispersion map), when the dispersion map is defocussing on average. The two solutions coexist for $\mu>\mu_{t h r}\left(D_{0}, m\right)$ and $E>E_{t h r}\left(D_{0}, m\right)$. Both the branches I and II correspond to a single branch $\mathrm{B}$ in the small-amplitude approximation under the normalization condition (15) [5].

In order to describe non-stationary dynamics of the Gaussian pulse near the periodic solutions, we derive a dynamical model from Eqs. (7) and (8) by setting,

$$
\alpha(z)=\alpha_{0}(\zeta)+\epsilon \alpha_{1}(z, \zeta)+\mathrm{O}\left(\epsilon^{2}\right)
$$

and

$$
\beta(z)=\int_{0}^{z} D\left(z^{\prime}\right) d z^{\prime}+\beta_{0}(\zeta)+\epsilon \beta_{1}(z, \zeta)+\mathrm{O}\left(\epsilon^{2}\right) .
$$

Here $\zeta=\epsilon z$ is the distance to measure the evolution of a Gaussian pulse over many map's periods. The coupled system (7) and (8) can be averaged over the map's period subject to the periodic conditions: $\alpha_{1}(z+1, \zeta)=\alpha_{1}(z, \zeta)$ and $\beta_{1}(z+1, \zeta)=\beta_{1}(z, \zeta)$. Then, the non-stationary system reduces to the dynamical model for $\alpha_{0}(\zeta)$ and $\beta(\zeta)$,

$$
\frac{d \alpha_{0}}{d \zeta}=F_{\alpha}\left(\alpha_{0}, \beta_{0}\right) \equiv \frac{E \alpha_{0}^{5 / 2}}{m}\left[\frac{1}{\left(\alpha_{0}^{2}+4 \beta_{0}^{2}\right)^{1 / 2}}-\frac{1}{\left(\alpha_{0}^{2}+4\left(\beta_{0}+m\right)^{2}\right)^{1 / 2}}\right],
$$




$$
\begin{aligned}
\frac{d \beta_{0}}{d \zeta}=F_{\beta}\left(\alpha_{0}, \beta_{0}\right) & \equiv D_{0}-\frac{E \alpha_{0}^{3 / 2}}{4 m}\left[\frac{4\left(m+\beta_{0}\right)}{\left(\alpha_{0}^{2}+4\left(m+\beta_{0}\right)^{2}\right)^{1 / 2}}-\frac{4 \beta_{0}}{\left(\alpha_{0}^{2}+4 \beta_{0}^{2}\right)^{1 / 2}}\right. \\
& \left.+\log \left(\frac{2 \beta_{0}+\left(\alpha_{0}+4 \beta_{0}^{2}\right)^{1 / 2}}{2\left(m+\beta_{0}\right)+\left(\alpha_{0}^{2}+4\left(m+\beta_{0}\right)^{2}\right)^{1 / 2}}\right)\right] .
\end{aligned}
$$

This system has of course the same stationary solutions $\alpha_{0}=\alpha_{s}$ and $\beta_{0}=\beta_{s}=-m / 2$ as those given in (13). The stationary solutions appear as equilibrium states in the dynamical system, whose stability can be found by linearizing,

$$
\begin{aligned}
& \alpha_{0}(\zeta)=\alpha_{s}+\Delta \alpha e^{i \lambda \zeta} \\
& \beta_{0}(\zeta)=\beta_{s}+\Delta \beta e^{i \lambda \zeta}
\end{aligned}
$$

where the eigenvalue $\lambda$ is

$$
\lambda^{2}(\mu)=-\frac{\partial F_{\alpha}}{\partial \beta}\left(\alpha_{s}\right) \frac{\partial F_{\beta}}{\partial \alpha}\left(\alpha_{s}\right)=\frac{2 E \alpha_{s}^{3 / 2}}{\left(m^{2}+\alpha_{s}^{2}\right)^{3 / 2}}\left[3 D_{0}+\frac{E \alpha_{s}^{3 / 2}\left(m^{2}-\alpha_{s}^{2}\right)}{\left(m^{2}+\alpha_{s}^{2}\right)^{3 / 2}}\right] .
$$

We plot $\lambda^{2}(\mu)$ in Fig.3 to confirm that $\lambda^{2}>0$ for branch I of the periodic solutions and $\lambda^{2}<0$ for branch II (cf. Fig. 2). Thus, the linear analysis predicts the instability of the short Gaussian pulses with larger energy at a fixed propagation constant $\mu$ (branch II). In the limit $\mu \rightarrow \mu_{t h r}\left(D_{0}, m\right)$, the instability disappears, i.e.

$$
\lim _{\mu \rightarrow \mu_{\text {thr }}} \lambda^{2}(\mu)=0 .
$$

This property follows from Eq. (17) in the limit $\mu \rightarrow \mu_{t h r}\left(D_{0}, m\right)$, when

$$
\frac{\partial F_{\beta}}{\partial E}\left(\alpha_{s}, \beta_{s}\right) \frac{d E}{d \mu}+\frac{\partial F_{\beta}}{\partial \alpha}\left(\alpha_{s}, \beta_{s}\right) \frac{d \alpha}{d \mu}=0
$$

Connecting Eqs. (18) and (19), we find the following asymptotic approximation,

$$
\lambda^{2} \rightarrow\left(\frac{\partial F_{\beta} / \partial E \cdot \partial F_{\alpha} / \partial \beta}{d \alpha / d \mu}\right) \frac{d E}{d \mu} .
$$

Taking into account that $d E / d \mu, \partial F_{\beta} / \partial E$, and $\partial F_{\alpha} / \partial \beta$ are all positive for $D_{0}<0$, and $\alpha \sim(\mu-$ $\left.\mu_{t h r}\right)^{1 / 2}$ (see Fig. 2(a,b)), the asymptotic approximation (20) produces the result, $\lambda \sim\left(\mu-\mu_{t h r}\right)^{1 / 4}$.

The nonlinear dynamics of the system (16) and (17) is shown in Fig. 4 for $D_{0}=-0.02$ and $m=2$. At a fixed value of the energy $E$, there are two stationary Gaussian pulses of different durations: a short pulse is a saddle point, while a long one is a center. Inside the separatrix loop, there are oscillations of the pulse trapped by the center point. Outside the separatrix, the Gaussian pulse transfers to chirped linear waves.

We notice that the transition scenario resembles the nonlinear dynamics of unstable solitons in generalized NLS equations [14]. The only difference is that the unstable branch in generalized 
(a)

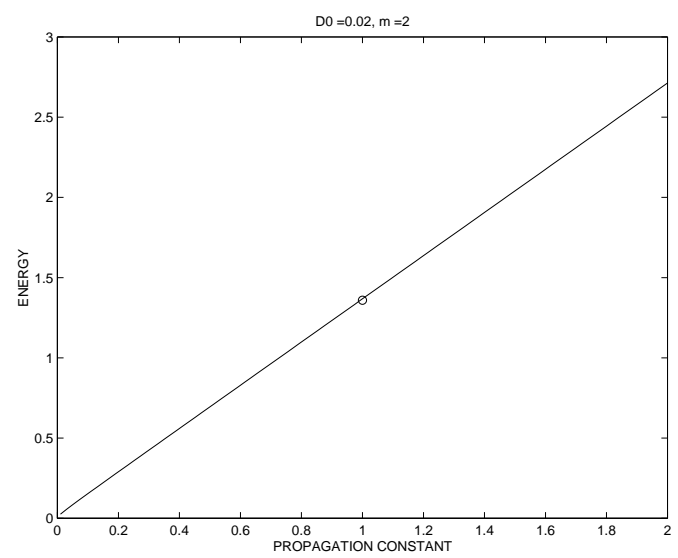

(b)

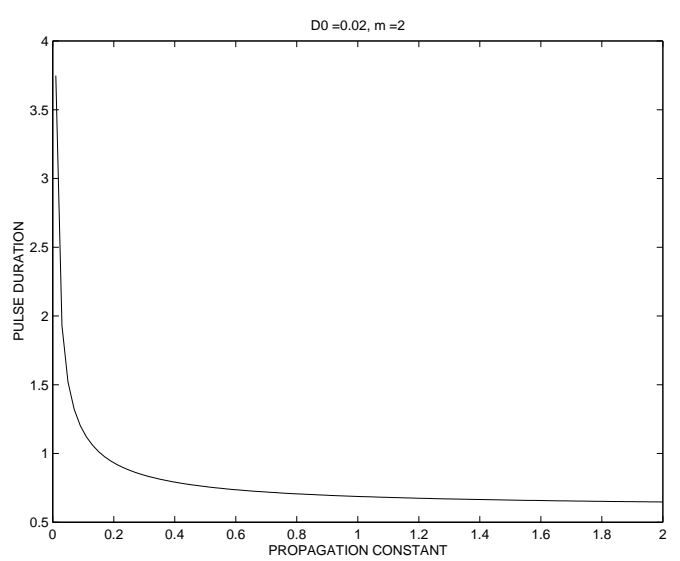

Figure 1: Energy $E$ (a) and pulse duration $\alpha_{s}$ (b) for the Gaussian pulse (6) at $D_{0}=0.02, m=2$.

(a)

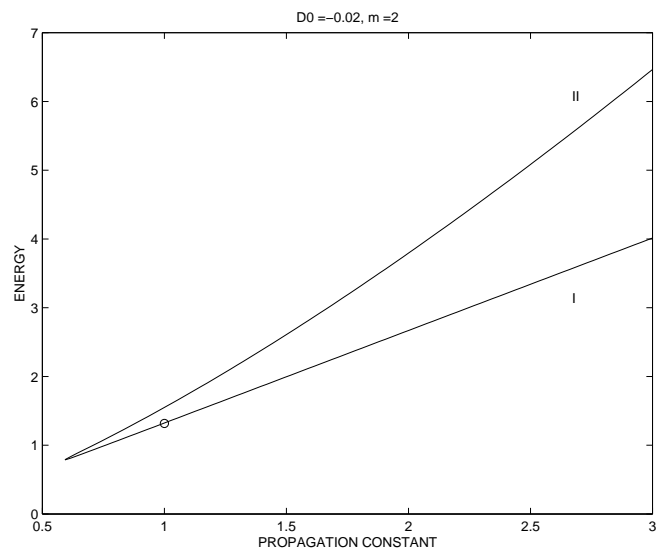

(b)

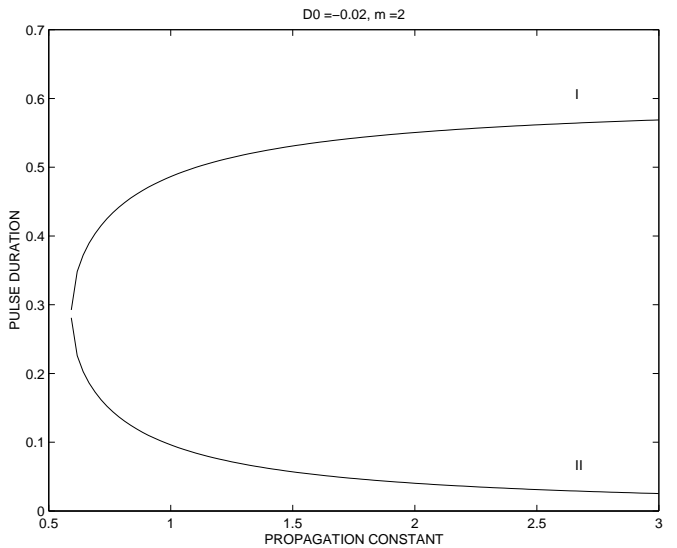

Figure 2: Energy $E$ (a) and pulse duration $\alpha_{s}$ (b) for the Gaussian pulse (6) at $D_{0}=-0.02, m=2$. 


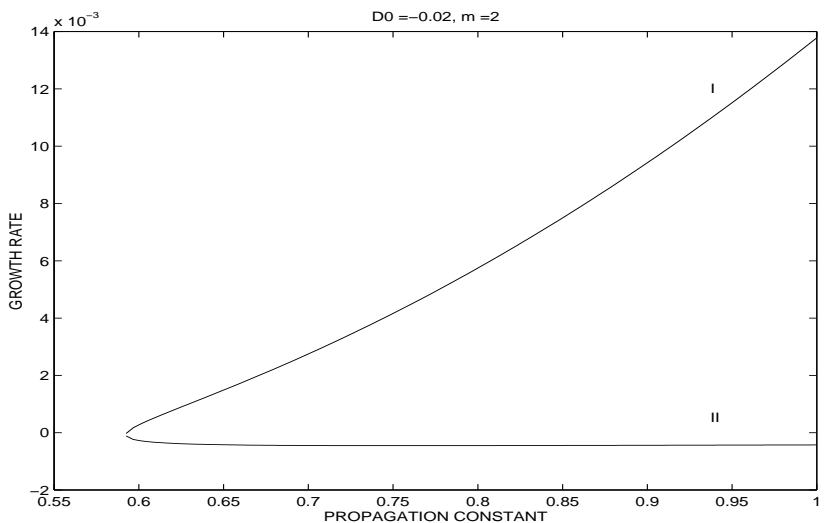

Figure 3: Growth rate $\lambda^{2}$ for the Gaussian pulse (6) at $D_{0}=-0.02, m=2$. The branch II with $\lambda^{2}<0$ corresponds to the unstable Gaussian pulse.

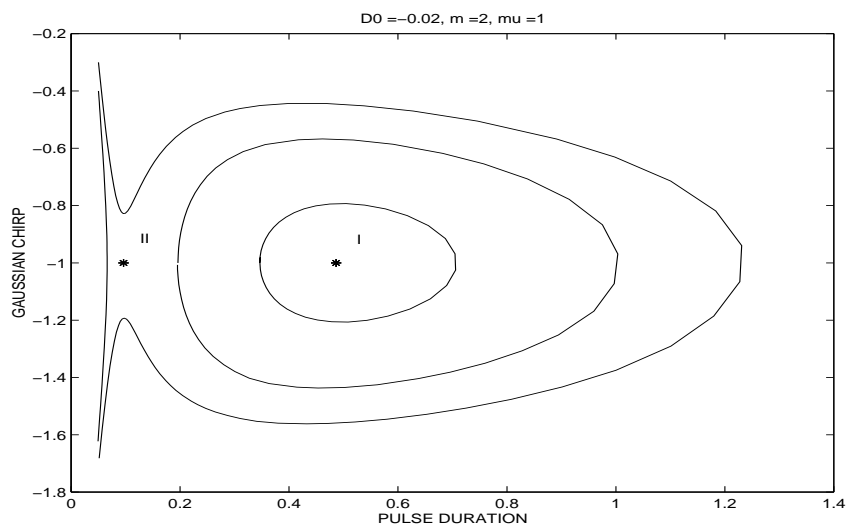

Figure 4: Phase plane $(\beta, \alpha)$ for the nonlinear dynamics of a Gaussian pulse (6) at $D_{0}=-0.02$, $m=2$ and $\mu=1$. 
NLS equations is located for those values of soliton propagation constant $\mu$, where $d E / d \mu<0$. Although this conventional stability criterion failed for the dispersion-managed solitons (see Fig. 2(a)), the instability development shows up to be alike (cf. Fig. 4 here and Fig. 2(b) in [14]).

\section{Integral evolution model: Numerical analysis}

The Gaussian approximation of the optical pulse in the NLS model (1) can be improved by summating all higher-order Gauss-Hermite solutions of the linear equation, $i u_{z}+0.5 D(z) u_{t t}=0$ as shown in [9]. However, this analysis results in a complicated infinite-dimensional system of algebraic equations for parameters and coefficients of the Gauss-Hermite expansion. Instead, we adopt a direct asymptotic method to deduce an integral evolution model valid in the limit $\epsilon \rightarrow 0$. This method is based on Fourier expansion of solutions of the linear equation above [10, 11] as well as on the asymptotic expansion,

$$
u(z, t)=u_{0}(z, t)+\epsilon u_{1}(z, t)+\mathrm{O}\left(\epsilon^{2}\right)
$$

where $u_{0}(z, t)$ is given in the Fourier form as

$$
u_{0}=\frac{1}{2 \pi} \int_{-\infty}^{\infty} d \omega W(\omega, \zeta) \exp \left(-\frac{i}{2} \omega^{2}\left(\int_{0}^{z} D\left(z^{\prime}\right) d z^{\prime}\right)+i \omega t\right) .
$$

Here $W(\omega, \zeta)$ is a complex Fourier coefficient and $\zeta=\epsilon z$ is the distance to measure the pulse evolution over many map's periods. By supplying the periodic condition $u_{1}(z+1, t)=u_{1}(z, t)$ in the Fourier form, the NLS equation (1) can be reduced to the integral evolution model,

$$
i W_{\zeta}-\frac{1}{2} D_{0} \omega^{2} W+\iint_{-\infty}^{\infty} d \omega_{1} d \omega_{2} r\left(\omega_{1} \omega_{2}\right) W\left(\omega+\omega_{1}\right) W\left(\omega+\omega_{2}\right) \bar{W}\left(\omega+\omega_{1}+\omega_{2}\right)=0,
$$

where

$$
r\left(\omega_{1} \omega_{2}\right)=\frac{1}{4 \pi^{2}} \int_{0}^{1} d z \exp \left(i \omega_{1} \omega_{2} \int_{0}^{z} D\left(z^{\prime}\right) d z^{\prime}\right) .
$$

For the two-step dispersion map (12), the integral kernel $r(x)$ can be computed explicitly as

$$
r(x)=\frac{1}{4 \pi^{2}} \frac{\sin \left(\frac{m x}{2}\right)}{\frac{m x}{2}} .
$$

It is obvious that the dynamical system (16) and (17) studied in the previous section can be deduced from (22) within the same Gaussian approximation. This correspondence implies that the qualitative results on instability of short Gaussian pulses for $D_{0}<0$ can be reconfirmed within a more systematic theory.

In this section we present numerical results consisting of three subsections. In the first subsection, we construct a numerical solution of the stationary problem identifying optical solitons 
in the normal regime, when $D_{0}<0$. In the second part, we analyze the linearized problem and locate numerically the linear spectrum in the problem, indicating possible instability of optical solitons. Then, we simulate numerically the non-stationary problem described by (22) and discuss the transformation routes of the unstable dispersion-managed solitons.

\subsection{Stationary solutions}

The periodic-type localized solutions of the NLS equation in the form (3) are equivalent to stationary solutions of (22) in the form,

$$
W(\omega, \zeta)=\Phi(\omega) e^{i \mu \zeta}
$$

where $\Phi(\omega)$ is the real Fourier coefficient which defines $\psi(z, t)$ according to (21). This function satisfies a nonlinear integral boundary-value problem,

$$
\left(\mu+\frac{1}{2} D_{0} \omega^{2}\right) \Phi(\omega)=R(\omega) \equiv \iint_{-\infty}^{\infty} d \omega_{1} d \omega_{2} r\left(\omega_{1} \omega_{2}\right) \Phi\left(\omega+\omega_{1}\right) \Phi\left(\omega+\omega_{2}\right) \Phi\left(\omega+\omega_{1}+\omega_{2}\right),
$$

where $\Phi(-\omega)=\Phi(\omega)$ (the symmetry condition) and $\lim _{\omega \rightarrow \infty} \Phi(\omega)=0$ (the boundary condition).

For numerical analysis, we intend to use the Petviashvili's iteration scheme [15:

$$
\Phi^{(n)}(\omega) \rightarrow \Phi^{(n+1)}(\omega)
$$

for $n=0,1,2, \ldots$ Within this scheme, the right-hand-side $R(\omega)$ can be approximated at the $n$-th approximation by $\Phi^{(n)}(\omega)$ provided a certain stabilizing factor is introduced for convergence (see (27) and (28) below). However, the numerical scheme breaks down for $D_{0}<0$ due to resonances at $\omega= \pm \omega_{\text {res }}$, where

$$
\omega_{\text {res }}=\sqrt{\frac{2 \mu}{\left|D_{0}\right|}} .
$$

Indeed for $\omega= \pm \omega_{\text {res }}$, the left-hand-side of (25) vanishes. [Here we notice that $\mu>0$ for the Gaussian pulse solutions ([6) of the NLS model (11).] In order to avoid resonances in the numerical scheme, we add and subtract a dummy positive dispersion term $0.5\left|D_{0}\right| \omega^{2} \Phi(\omega)$ to the left-hand-side of (25). As a result, the scheme converts to the following map,

$$
\Phi^{(n)}(\omega) \rightarrow \Phi^{(n+1)}(\omega)=S_{n}^{3 / 2}\left(\frac{R^{(n)}(\omega)+\frac{1}{2}\left(\left|D_{0}\right|-D_{0}\right) \omega^{2} \Phi^{(n)}(\omega)}{\mu+\frac{1}{2}\left|D_{0}\right| \omega^{2}}\right), \quad D_{0}<0,
$$

where $S_{n}$ is the Petviashvili's stabilizing factor given by

$$
S_{n}=\frac{\int_{-\infty}^{\infty} d \omega\left(\mu+\frac{1}{2}\left|D_{0}\right| \omega^{2}\right) \Phi^{(n)}(\omega)}{\int_{-\infty}^{\infty} d \omega \Phi^{(n)}(\omega)\left(R^{(n)}(\omega)+\frac{1}{2}\left(\left|D_{0}\right|-D_{0}\right) \omega^{2} \Phi^{(n)}(\omega)\right)}
$$


The factor $S_{n}$ is unity at the stationary solution and serves therefore as an indicator for termination of the iterating procedure. We stop iterations when $\left|S_{n}-1\right|<10^{-5}$.

To use the map (27), we apply the Simpson's integration method, reducing complexity due to the symmetry: $\Phi(-\omega)=\Phi(\omega)$. As a starting solution, the profile $\Phi(\omega)$ can be approximated by the Gaussian pulse with parameters corresponding to the periodic solution (13) and (14),

$$
\Phi^{(0)}(\omega)=\sqrt{\pi E \sqrt{2 \alpha_{s}}} \exp \left(-\frac{1}{4} \alpha_{s} \omega^{2}\right) .
$$

\begin{tabular}{|l|l|l|l|}
\hline Number of iterations & $S_{n}: D_{0}=0.02$ & $S_{n}: D_{0}=-0.02(\mathrm{I})$ & $S_{n}: D_{0}=-0.02(\mathrm{II})$ \\
\hline 1 & 0.9897 & 0.9957 & 0.9920 \\
\hline 2 & 0.9971 & 0.9981 & 0.9917 \\
\hline 3 & 0.9994 & 0.9988 & 0.9921 \\
\hline 4 & 0.9998 & 0.9991 & 0.9942 \\
\hline 5 & 0.9999 & 0.9993 & 0.9989 \\
\hline 6 & & 0.9994 & 1.0069 \\
\hline 7 & & 0.9995 & 1.0167 \\
\hline 8 & & 0.9996 & 1.0173 \\
\hline 9 & & 0.9997 & 0.9642 \\
\hline 10 & & 0.9997 & 0.8133 \\
\hline 11 & & 0.9998 & 0.7025 \\
\hline 12 & & 0.9998 & 0.6833 \\
\hline 13 & & 0.9999 & 0.6773 \\
\hline 14 & & 0.9999 & 0.6695 \\
\hline 15 & & 0.9999 & 0.6601 \\
\hline 16 & & & 0.6499 \\
\hline 17 & & & 0.6394 \\
\hline 18 & & & 0.6292 \\
\hline 19 & & & 0.6192 \\
\hline
\end{tabular}

Table I. Iterations of the stabilizing factor $S_{n}$ for $D_{0}=0.02$ and $D_{0}=-0.02$ (branches I and II).

Table 1 shows iterations for the stabilizing factor $S_{n}$ in the three different cases: (i) $D_{0}=0.02$, (ii) $D_{0}=-0.02$ (branch I), and (iii) $D_{0}=-0.02$ (branch II). For all the cases, the other parameters are $\mu=1$ and $m=2$. In the first case, the convergence is monotonic and the profile for stationary soliton $\Phi(\omega)$ is shown in Fig. 5(a). The numerical value for energy of the stationary soliton is shown in Fig. 1(a) by a bullet. In the second case, the iterations converge slowly to the stationary soliton shown in Fig. 5(b). Sometimes, the convergence is accompanied by a single oscillation of $S_{n}$ near unity. The numerical value for the energy is shown in Fig. 2(a) by a bullet at branch I. In the last case, however, the iterations oscillate and finally diverge. Inspection of the profile $\Phi^{(n)}(\omega)$ at a final iteration shows that the iterations change the initial pulse drastically leading to its 

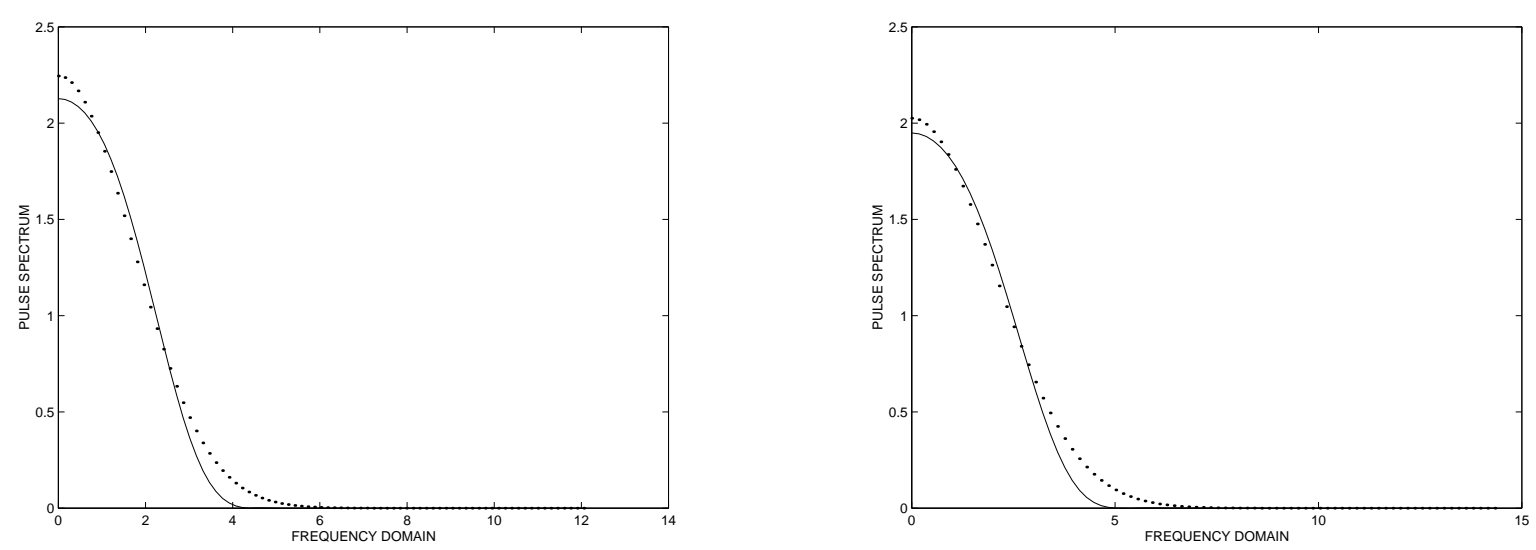

Figure 5: The profile of the stationary pulse $\Phi(\omega)$ in the boundary-value problem (25) at $D_{0}=0.02$ (a) and $D_{0}=-0.02$ (branch I) (b). The other parameters are: $m=2$ and $\mu=1$. The dotted line displays the Gaussian pulse (29) for the same parameter values.

disappearance. Two conjectures follow from this fact. Either the shorter soliton with larger energy at branch II does not exist as a stationary solution of (25) or it is unstable within the iterational scheme (27). Since the short Gaussian pulse does exist (see Fig. 2), we incline to work along the second conjecture. The iterational scheme (27) is not relevant for the time-evolution problem and rigorous analysis of linearized problem is needed to confirm predictions of the instability of the short stationary pulse.

\subsection{Linear spectrum}

There are several forms of the linear problem associated to the NLS-type equations. We will use the matrix form which was studied in our previous paper [16] subject to certain simplifications. The matrix form appears upon perturbations of the stationary solutions of (22) as

$$
W(\omega, \zeta)=e^{i \mu \zeta}\left[\Phi(\omega)+w_{1}(\omega) e^{i \lambda \zeta}+\bar{w}_{2}(\omega) e^{-i \lambda \zeta}\right]
$$

The vector $\mathbf{w}(\omega)=\left(w_{1}, w_{2}\right)^{T}$ can be shown to satisfy the matrix linear problem,

$$
\lambda \mathbf{w}(\omega)=-\left(\mu+\frac{1}{2} D_{0} \omega^{2}\right) \sigma_{3} \mathbf{w}(\omega)+\int_{-\infty}^{\infty} d \omega_{1}\left(2 K_{1}\left(\omega, \omega_{1}\right) \sigma_{3}+K_{2}\left(\omega, \omega_{1}\right) \sigma_{2}\right) \mathbf{w}\left(\omega_{1}\right),
$$

where

$$
\sigma_{3}=\left[\begin{array}{cc}
1 & 0 \\
0 & -1
\end{array}\right], \quad \sigma_{2}=\left[\begin{array}{cc}
0 & 1 \\
-1 & 0
\end{array}\right]
$$



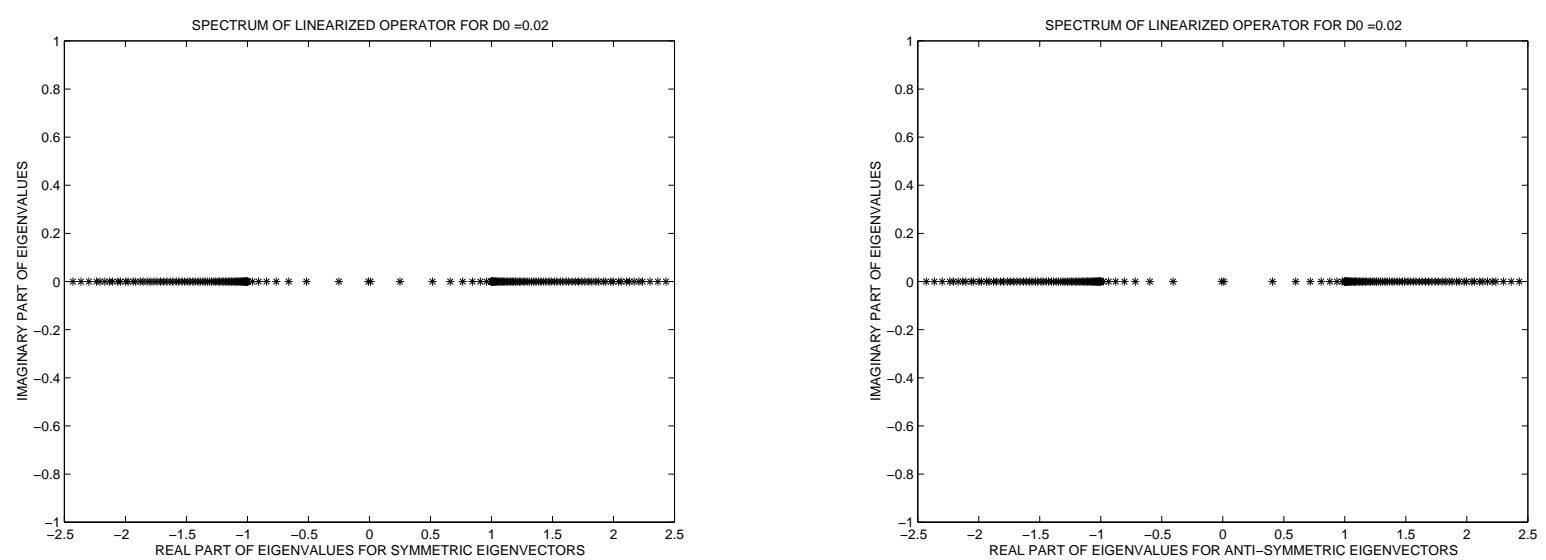

Figure 6: (a,b) The linear spectrum $\lambda$ of Eq. (31) for the stationary pulse at $D_{0}=0.02, m=2$ and $\mu=1$ which corresponds to Fig. 5(a).

and the integral kernels are

$$
\begin{aligned}
& K_{1}\left(\omega, \omega_{1}\right)=\int_{-\infty}^{\infty} d \omega_{2} r\left[\left(\omega-\omega_{1}\right)\left(\omega-\omega_{2}\right)\right] \Phi\left(\omega_{2}\right) \Phi\left(\omega_{1}+\omega_{2}-\omega\right), \\
& K_{2}\left(\omega, \omega_{1}\right)=\int_{-\infty}^{\infty} d \omega_{2} r\left[\left(\omega_{2}-\omega_{1}\right)\left(\omega_{2}-\omega\right)\right] \Phi\left(\omega_{2}\right) \Phi\left(\omega_{1}-\omega_{2}+\omega\right) .
\end{aligned}
$$

Using the stationary solution $\Phi(\omega)$ from the previous subsection and implementing the Simpson's integration method again, we solve the linear problem by using the linear algebra packages built in Matlab 5.2. We identify two types of modes of the linear spectrum: symmetric eigenfunctions, when $\mathbf{w}(-\omega)=\mathbf{w}(\omega)$, and anti-symmetric eigenfunctions, when $\mathbf{w}(-\omega)=-\mathbf{w}(\omega)$.

The linear spectrum for the soliton of Fig. 5(a) is shown in Fig. 6(a,b). It consists of three main parts: (i) continuous spectrum, (ii) neutral (zero) modes, and (iii) internal (oscillatory) modes.

When $D_{0}>0$ (the anomalous regime of the dispersion map), the continuous spectrum is located at the real axis for $|\lambda|>\mu$ (see Fig. 6(a,b) where $\mu=1$ ). Indeed, the continuous modes are singular in the Fourier representation, i.e. $\mathbf{w}(\omega) \sim \delta(\omega-\Omega)$. Then, the linear problem (31) has the continuous spectrum at $\lambda= \pm \lambda_{\Omega}$, where

$$
\lambda_{\Omega}=\mu+\frac{1}{2} D_{0} \Omega^{2},
$$

provided the following integral kernels are not singular,

$$
\begin{aligned}
\lim _{\omega \rightarrow \Omega} K_{1}(\omega, \Omega-\omega) & =\frac{1}{4 \pi^{2}} \int_{-\infty}^{\infty} d \omega \Phi^{2}(\omega), \\
\lim _{\omega \rightarrow \Omega} K_{2}(\omega, \Omega-\omega) & =\int_{-\infty}^{\infty} d \omega r\left(\omega^{2}\right) \Phi(\omega+\Omega) \Phi(\omega-\Omega) .
\end{aligned}
$$



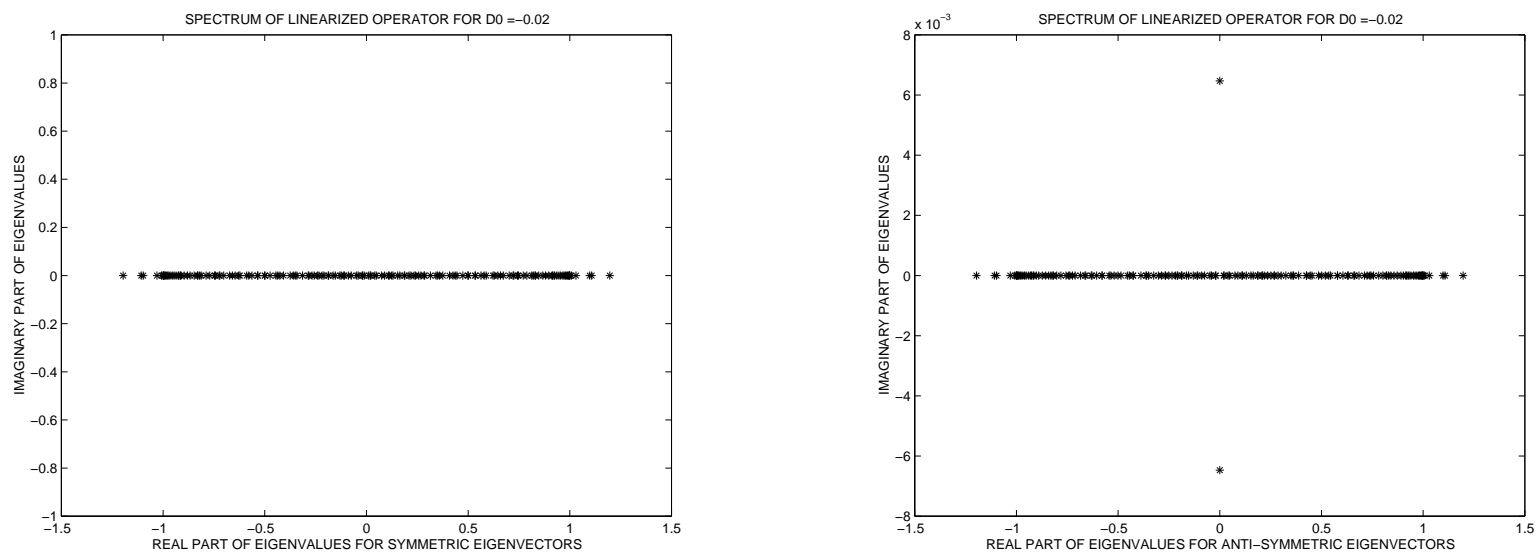

Figure 7: (a,b) The linear spectrum $\lambda$ of Eq. (31) for the stationary pulse at $D_{0}=-0.02, m=2$ and $\mu=1$ which corresponds to Fig. 5(b).

The neutral (zero) modes always appear at $\lambda=0$ as double degenerate states for both symmetric and asymmetric eigenfunctions. However, the inaccuracy of the numerical method destroys the degeneracy of the zero modes. As a result, the two zero modes may appear either for small real or for small imaginary values of $\lambda$. Fig. 7(b) displays two imaginary eigenvalues of order of $\mathrm{O}\left(10^{-2}\right)$ which appear to be shifted from the origin of $\lambda$ due to this numerical effect. Since the zero modes are not of interest from stability point of view, we neglect this effect and leave the scheme without any additional modification.

The internal (oscillatory) modes are located in the gap of the continuous spectrum as $0<|\lambda|<$ $\mu$. The set of internal modes may contain different number of eigenvalues. We have shown in previous paper [16] that the set is empty in the NLS limit (which corresponds to the limit $\mu \rightarrow 0$ at fixed $D_{0}>0$ and $m$ ). [Note that in [16] we used the Gaussian pulse (29) for approximating $\Phi(\omega)$ while here we substitute the numerical result from Eq. (25).] Then, we showed that the number of internal modes increases with the map's strength (if the parameter $\alpha_{s}$ is set to unity, the map's strength is proportional to $m$ and vice versa). In Fig. $6(\mathrm{a}, \mathrm{b})$ for $\mu=1$, we identify 14 internal modes for symmetric eigenfunctions and 12 internal modes for anti-symmetric eigenfunctions. Still complex eigenvalues are absent for $D_{0}>0$ which confirms stability of dispersion-managed solitons in the anomalous regime.

The linear spectrum for the soliton of Fig. 5(b) is shown in Fig. 7(a,b). The continuous spectrum is seen to have changed drastically. When $D_{0}<0$ (normal regime of the dispersion map), 
(a)

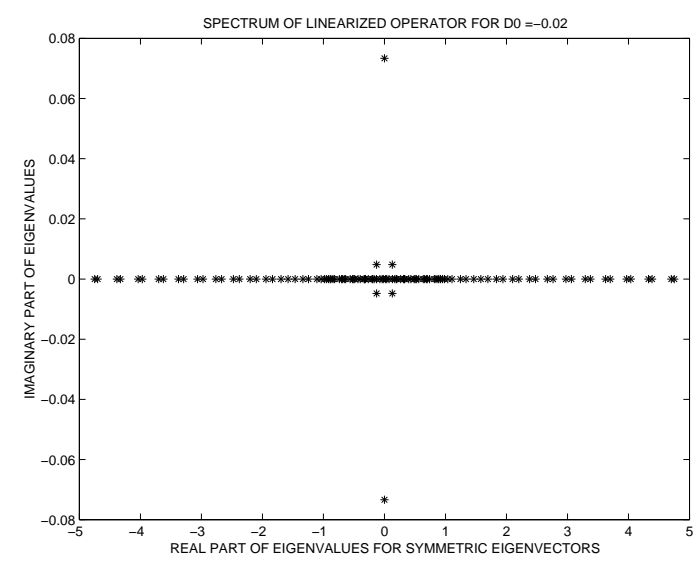

(c)

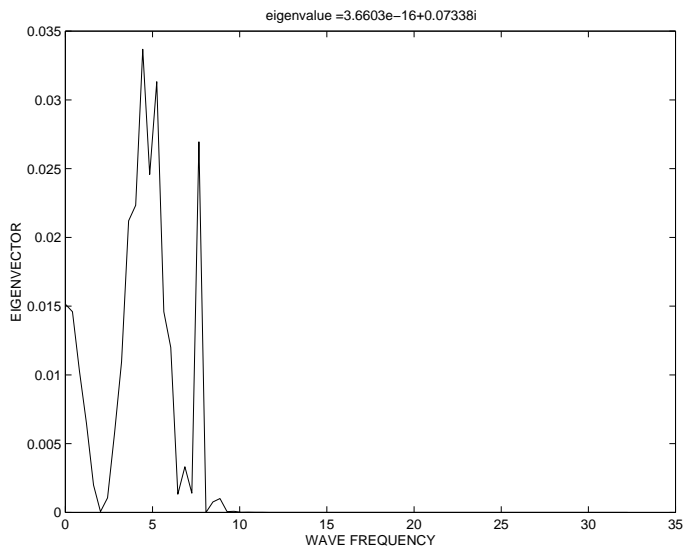

(b)

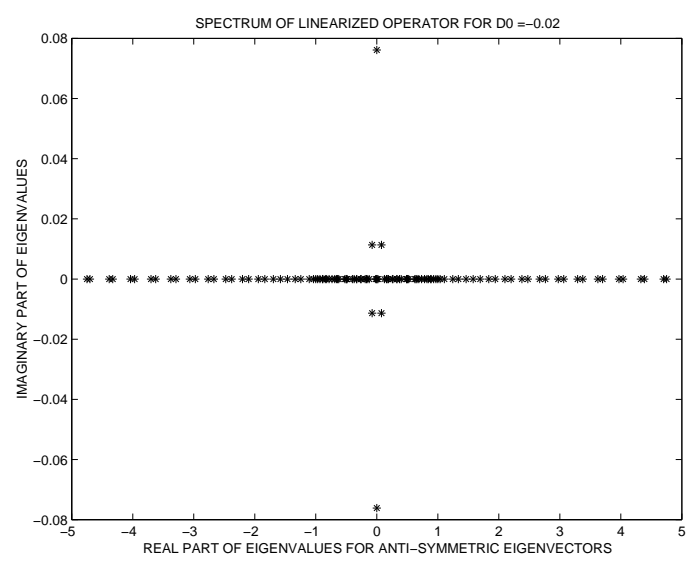

(d)

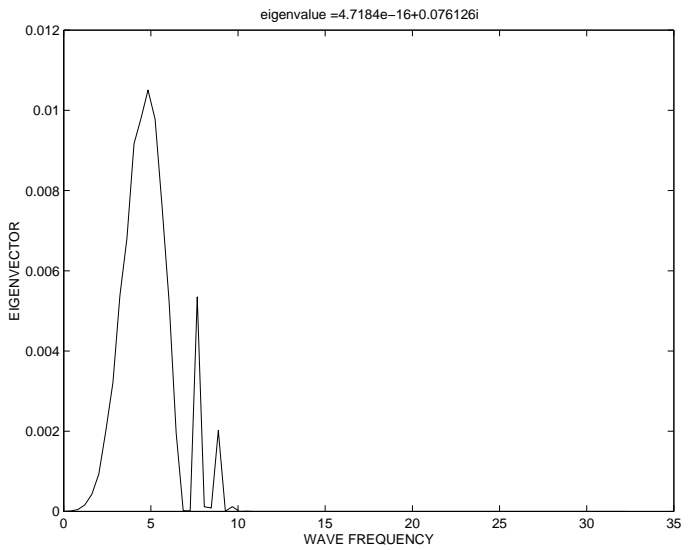

Figure 8: (a,b) The linear spectrum $\lambda$ of Eq. (31) for the stationary pulse at $D_{0}=-0.02, m=2$ and $\mu=1$ which corresponds to the Gaussian pulse (6) at branch II. (c,d) The symmetric (c) and antisymmetric $(d)$ eigenvectors $\mathbf{w}(\omega)$ for the unstable eigenvalues with $\operatorname{Im}(\lambda)>0$. 
the continuous spectrum covers the segment $|\lambda|<\mu$ twice according to (32). As a result, neutral and internal modes, if any, become embedded in the wave continuum as seen in Fig. 7(a,b). This indicates a resonance of stationary soliton with the linear spectrum in the normal regime of the dispersion map. However, this resonance does not result to any instability of solitons of branch I within the linear theory. We discuss the resonance issue in Section 4. The two imaginary eigenvalues on Fig. 7(b) appear from the origin as artifacts of the numerical scheme as it was explained above.

At last, we would like to construct the linear spectrum for the solitons of branch II. However, the stationary solutions were not identified within the Petviashvili's numerical method. Therefore, assuming that the solutions still exist, the profile $\Phi(k)$ can only be approximated by the Gaussian pulse (29) as we did in [16]. The linear spectrum in this approximation is shown in Fig. 8(a,b) for $D_{0}=-0.02, m=2$, and $\mu=1$. The same type of the continuous spectrum is clearly seen not to possess any gap in the origin. In addition to this, we identify new complex eigenvalues both for symmetric and anti-symmetric eigenfunctions. These complex eigenvalues has relatively large, order of $\mathrm{O}\left(10^{-1}\right)$, imaginary part and they are associated with the instability of the solitons of branch II. The numerical result for the instability eigenvalues is in a reasonable comparison with the asymptotic predictions which follow from the dynamical model (16) and (17) (see Fig. 3, branch II). The eigenvectors $\mathbf{w}(\omega)$ for the unstable (imaginary) eigenvalues are shown in Fig. 8(c,d) for

the symmetric and anti-symmetric eigenfunctions, respectively. The numerical approximations of the eigenvectors, being inaccurate in details, display clearly that the unstable modes are localized at the intermediate wave frequencies $\omega$ of the pulse spectrum (at $\omega \approx 4.5$ ). Thus, the development of the unstable eigenvectors would affect the duration of the soliton pulse in the nonlinear stage as described in the next subsection.

\subsection{Non-stationary evolution}

To confirm the transition scenario, we simulate the non-stationary dynamics of unstable solitons in the integral model (22) by using the central-difference scheme,

$\frac{V^{(n+1)}(\omega)-V^{(n-1)}(\omega)}{2 \Delta \zeta}=i \iint_{-\infty}^{\infty} d \omega_{1} d \omega_{2} r\left(\omega_{1} \omega_{2}\right) e^{i D_{0} \omega_{1} \omega_{2} \zeta_{n}} V^{(n)}\left(\omega+\omega_{1}\right) V^{(n)}\left(\omega+\omega_{2}\right) \bar{V}^{(n)}\left(\omega+\omega_{1}+\omega_{2}\right)$

where

$$
V^{(n)}(\omega)=W\left(\omega, \zeta_{n}\right) \exp \left(\frac{i}{2} D_{0} \omega^{2} \zeta_{n}\right)
$$

and $\zeta_{n}=n \Delta \zeta$. An initial iteration can be done within a forward scheme starting with the initial Gaussian pulse (29) with the parameter $\alpha_{s}$ and $E$ corresponding to branches I and II at $\mu=1$ on 
Fig. 2(a,b). Evolution of a stable long pulse (branch I) is shown in Fig. 9(a,b), while that of an unstable short pulse (branch II) is shown in Fig. 9(c,d). The stable long Gaussian pulse quickly transits to the stationary pulse given by Eq. (24) (Fig. 5(b)) which propagates later without visible distortions. The second and third peeks in the signal spectrum and profile (Fig. 9(a,b)) appear in complete agreement with the profile of the stationary DM soliton (cf. Fig. 1 from [11]). Some oscillations along the distance $\zeta$ are excited due to the difference between the Gaussian pulse (29) and the exact stationary solution of Eq. (25). These oscillations are small compared to the soliton profile and they do not change the duration of the soliton pulse (see Fig. 9(a)). The nonlinear resonance at $\omega_{\text {res }}\left(\omega_{\text {res }}=10\right.$ for Fig. $\left.9(\mathrm{a})\right)$ is not seen to be excited during the signal propagation.

Evolution of the short (unstable) pulse differs drastically from the previous picture. The pulse is being broaden during the evolution, it generates the strong radiation tail and tends to the long (stable) signal which has the first node at $\omega \approx 4.5$ (cf. Fig. 9(a) and Fig. 9(c)). This transformation is accompanied by the intermediate oscillations around the soliton's shape. Thus, we confirm the analytical predictions that the short pulses are linearly unstable and switch into long stable solitons via long-term oscillatory dynamics. The unstable eigenvectors at Fig. 8(c,d) clearly match at Fig. 9 (c) with the growing deformations of the localization of the pulse spectrum.

\section{Discussion: Resonance of dispersion-managed solitons}

We have shown that both the Gaussian variational approximation and the integral model prescribe the instability of short nonlinear signals in the normal regime of the dispersion map. This instability broadens the signal's profile through some intermediate oscillations. The long signals propagate stably then.

We point out that the small-amplitude approximation considered here corresponds to the asymptotic limit $E / D_{0} \rightarrow 0$ (see branch B at Fig. 1 in [5]). The stability of the small-amplitude branch for $D_{0}<0$ was previously under discussion in the literature [3, 5, 12]. This discussion can be resolved if one takes into account the normalization condition (15), which was used in the previous works. We have checked that the normalization condition (15) selects the pulse solution along the stable branch I for $S_{t h r}<S<S_{\text {stab }}$, where $S_{\text {stab }} \approx 6.76$. For $S>S_{\text {stab }}$, it selects solutions along the unstable branch II (see Figs. 2(a,b)). Thus, for the intermediate map strengths (when $S<S_{\text {stab }}$ ),

the soliton signal propagates stably in the limit of small energies, as reported in [5]. However, if the map strength $S$ exceeds the value $S_{s t a b}$, the soliton signal breaks down and switches to a longer signal, as conjectured in [3]. 
(a)

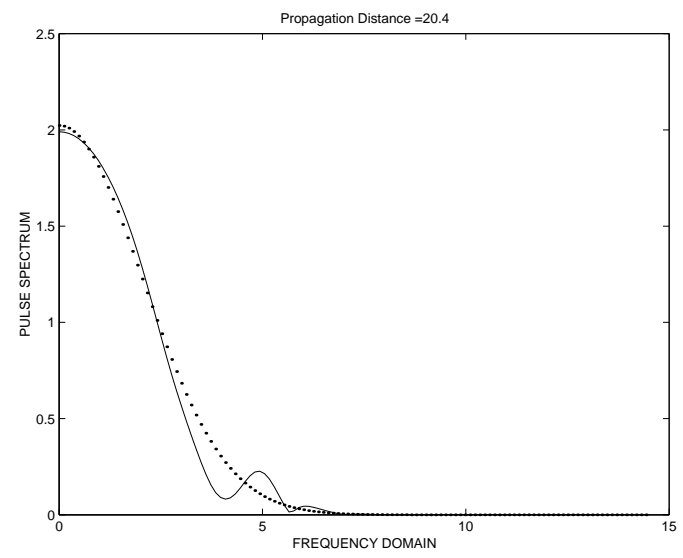

(c)

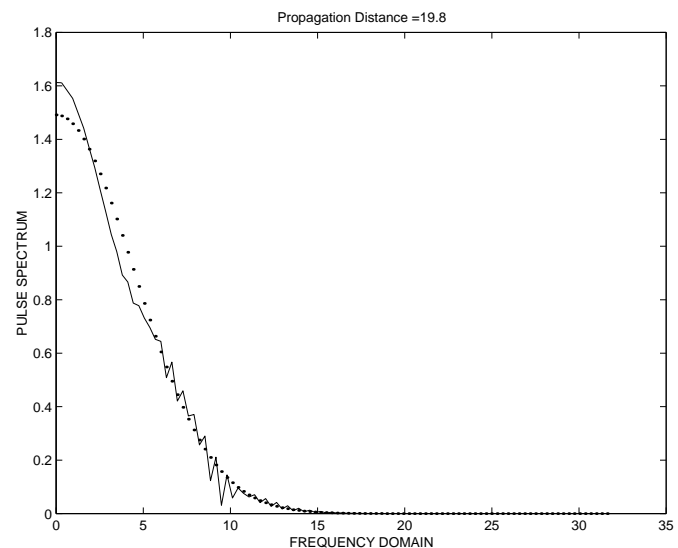

(b)

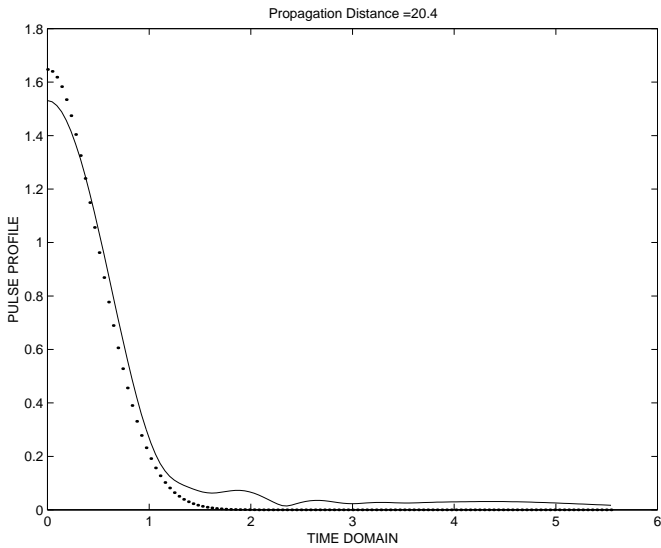

(d)

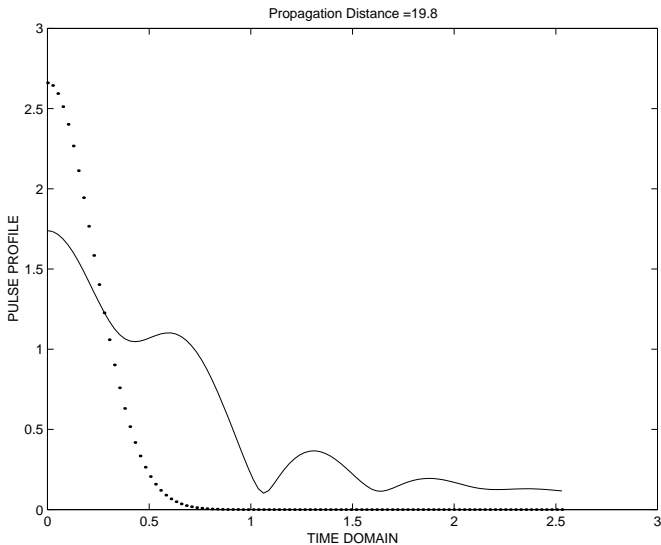

Figure 9: Propagation of the stable $(\mathrm{a}, \mathrm{b})$ and unstable $(\mathrm{c}, \mathrm{d})$ Gaussian pulses in the model (22) at $D_{0}=-0.02$ and $m=2$. The dotted line displays the initial pulse (29) for the branch I (a,b) and for the branch II $(\mathrm{c}, \mathrm{d})$ at $\mu=1$. The solid line displays the profile at $\zeta=20$. 
For both the branches, we observe the resonance appearing between the linear wave spectrum and the stationary signals. This resonance is related to the fact that the origin $\lambda=0$ is absorbed in the continuous spectrum of the linear problem. Another way to find the resonance is to construct the linear spectrum for the integral model (22), $W(\omega, \zeta) \sim W_{0} e^{i \Omega(\omega) \zeta}$, where

$$
\Omega=-\frac{1}{2} D_{0} \omega^{2} \geq 0
$$

and $D_{0}<0$. Therefore, for any $\mu>0$ there exists $\omega_{\text {res }}$ such that $\Omega\left(\omega_{\text {res }}\right)=\mu$. This resonance does not show up in the linear theory since the discrete and continuous spectra are separated. However, in the nonlinear stage, the resonance generally leads to emission of wave packets and soliton's decay.

Since the transformation or decay of long stable solitons for $D_{0}<0$ have never been observed numerically (nor in our simulations reported in Figs. 9), it is likely that the effective gap in the spectrum still appears in the nonlinear theory. Also the truncated approximation given by the integral model (22) may not be valid for a correct description of the resonance. The latter issues remain open for further analytical consideration.

\section{Aknowledgements.}

The author thanks S. Turitsyn and G. Biondini for bringing the present problem to his attention as well as T. Lakoba and C. Sulem for collaboration and valuable remarks. Fig. 9 was prepared by using computational capacities of Institute of Optics at Rochester, NY.

\section{References}

[1] N.J. Smith, N.J. Doran, W. Forysiak, and F.M. Knox, J. Lightwave Technol. 15, 1808 (1997).

[2] S.K. Turitsyn, V.K. Mezentsev, and E.G. Shapiro, Opt. Fiber Technol. 4, 384 (1998).

[3] A. Berntson, N.J. Doran, W. Forysiak, and J.H.B. Nijhof, Opt. Lett. 23, 900 (1998).

[4] J.H.B. Nijhof, N.J. Doran, and W. Forysiak, Opt. Lett. 23, 1674 (1998).

[5] V.S. Grigoryan and C.R. Menyuk, Opt. Lett. 23, 609 (1998).

[6] T.I. Lakoba, J. Yang, D.J. Kaup, and B.A. Malomed, Opt. Comm. 149, 366 (1998).

[7] S.K. Turitsyn, A.B. Aceves, C.K.R.T. Jones, and V. Zharnitsky, Phys. Rev. E 58, 48 (1998).

[8] S.K. Turitsyn and E.G. Shapiro, Opt. Fiber Technol. 4, 151 (1998). 
[9] T.I. Lakoba and D.J. Kaup, Phys. Rev. E 58, 6728 (1998).

[10] I. Gabitov and S.K. Turitsyn, Opt. Lett. 21, 327 (1996).

[11] M.J. Ablowitz and G. Biondini, Opt. Lett. 23, 1668 (1998).

[12] S.K. Turitsyn and V.K. Mezentsev, JETP Lett. 68, 640 (1998).

[13] C. Pare, V. Roy, F. Lesage, P. Mathieu, and P.-A. Belanger, Phys. Rev. E 60, 4836 (1999).

[14] D.E. Pelinovsky, V.V. Afanasjev, and Yu.S. Kivshar, Phys. Rev. E 53, 1940 (1996).

[15] V.I. Petviashvili and O.A. Pokhotelov, Solitary Waves in Plasmas and in the Atmosphere (Gordon and Breach, London, 1992), p.264.

[16] T.I. Lakoba and D.E. Pelinovsky, Chaos, to be published (2000). 